\title{
Diferencias de sexo y edad en el autoconcepto social ${ }^{*}$
}

\section{Sex and age differences in social self-concept}

Recepción: 28 Octubre 2014 | Aprobación: 25 Enero 2017

\author{
Arantza Fernández Zabala ${ }^{\mathrm{a}}$ \\ Universidad del País Vasco, España \\ ORCID: http://orcid.org/0000-0001-8528-1558 \\ Eider GoÑ I Palacios \\ Universidad del País Vasco, España \\ Arantzazu Rodríguez Fernández \\ Universidad del País Vasco, España \\ Alfredo Goñi Grandmontagne \\ Universidad del País Vasco, España
}

a Autor de correspondencia. Correo electrónico: arantza.fernandez@ehu.es

\section{RESUMEN}

Este estudio tiene por objeto identificar diferencias asociadas a la edad y al sexo en el autoconcepto social medido con el Cuestionario de Autoconcepto Social (AUSO), de Fernández-Zabala (2011), que consta de las escalas de responsabilidad social y de competencia social. Participaron en la investigación 767 estudiantes, 318 hombres (41.5\%) y 449 mujeres (58.5\%), de entre 16 y 25 años $(\mathrm{M}=19.97$; DT $=1.86)$ divididos en dos grupos de edad (menores de 20 años y mayores de 20). Tras comprobar la invarianza factorial del cuestionario por sexo y edad, se verifican diferencias en la escala de responsabilidad social, y no en la de competencia social, a favor de las mujeres y del grupo de más edad. Estos resultados suponen un avance respecto de anteriores conocimientos sobre el desarrollo del autoconcepto social a la par que refrendan la calidad psicométrica del AUSO.

\section{Palabras clave}

Autoconcepto social; instrumento de medida; invarianza factorial; sexo; edad

Para citar: Fernández, A., Goñi, E., Rodríguez, A., \& Goñi, A. (2017). Diferencias de sexo y edad en el autoconcepto social. Universitas psychologica 16(2), 1-10.

https://doi.org/10.11144/Javeriana.upsy.16-2.d sea

\begin{abstract}
The aim of this study is to identify differences associated with age and sex in the social self-concept measured by the Social Self-Concept Questionnaire (AUSO), by Fernández-Zabala (2011), made up for two scales: social responsibility and social competence. In the research participated a total of 767 students, 318 men (41.5\%) and 449 women (58.5\%), between 16 and 25 years $(\mathrm{M}=19.97, \mathrm{SD}=1.86)$ divided into two age groups (under 20 years and older than 20). After checking the factorial invariance of the questionnaire by sex and age, differences in the scale of social responsibility are verified, but not on social competence, in favor of women and older group. These results represent an advance over previous knowledge on the development of social self-concept and at the same time endorse AUSO psychometric quality.

Keywords

Social self-concept; questionnaire; factorial invariance; sex; age
\end{abstract}

| Universitas Psychologica | Colombia | V. i6 | No. 2 | Abril-Junio | 20 I 7 | ISSN i657-9267 | 


\section{Introducción}

Desde la década de los setenta del pasado siglo se ha venido prestando considerable atención en las investigaciones psicológicas al estudio no sólo del autoconcepto general, en cuanto autopercepción global, sino a otras autopercepciones de menor amplitud (Peixoto \& Almeida, 2011; Shavelson, Hubner, \& Stanton, 1976) tales como el autoconcepto físico (Esnaola, Infante, \& Zulaika, 2011; Goñi, Rodríguez, \& Esnaola, 2010; Rodríguez-Fernández, Axpe, \& Goñi, 2015), el autoconcepto académico (Xu et al, 2013), el autoconcepto personal (Goñi, Madariaga, Axpe, \& Goñi, 2011) y el autoconcepto social (Fernández-Zabala, Rodríguez-Fernández, \& Goñi, 2016).

Lo investigado sobre el autoconcepto social en particular no es poco y sin embargo subsisten interrogantes sobre aspectos tan básicos del mismo, como su asociación con la edad y con el sexo.

No se verifican diferencias asociadas al sexo en las respuestas dadas al cuestionario de Harter (Chang, McBride-Chang, Stewart, \& Au, 2003; Harter, 1982; Pastor, Balaguer, \& GarcíaMerita, 2003) ni al de Bracken (1992), así como tampoco en la escala de autoconcepto social (Amezcua \& Pichardo, 2000; Costa \& Tabernero, 2012; Garaigordobil, Dura, \& Pérez, 2005) de los cuestionarios Autoconcepto FormaA (AFA) de Musitu, García y Gutiérrez (1991) y Autoconcepto Forma-5 (AF-5) de García y Musitu (2001). En cambio, en las respuestas al Self Description Questionnaire (versiones II y III), en unos casos las mujeres muestran una mejor autopercepción que los varones de sus relaciones con personas del mismo sexo (Inglés, Pastor, Torregrosa, Redondo, \& GarcíaFernández, 2009; Marsh, 1989), y en algún caso (Bowles \& Fallon, 1996) las diferencias no resultan significativas. Por otro lado, los varones logran puntuaciones más altas que las mujeres en la autopercepción de las relaciones con personas del sexo opuesto (Marsh, 1989), aunque en otros estudios no se constatan diferencias significativas entre uno y otro sexo (Bowles \& Fallon, 1996; Inglés et al., 2009). Puede concluirse que, en general, no se han comprobado diferencias asociadas al sexo salvo en la autopercepción de las relaciones con personas del mismo sexo y del sexo opuesto donde los datos no son concluyentes.

Con respecto a la edad, las respuestas dadas a los cuestionarios AFA indican un inicio de descenso en el autoconcepto social a partir del Bachillerato (Esnaola, 2005; García \& Musitu, 2001), mientras que en la escala de relaciones con el sexo opuesto de los SDQ se ha comprobado un incremento con la edad, entre los 12 y los 16 años (Inglés et al., 2009), y un descenso de la autopercepción de las relaciones con iguales del mismo sexo después de los 15 años (Marsh, 1989). Tampoco el balance con respecto a las diferencias en el autoconcepto social asociadas a la edad ofrece abundancia de datos ni resultados concluyentes.

Llama, además, poderosamente la atención que se pretenda explicar el autoconcepto social, a nuestro modo de ver de forma reduccionista, como un conjunto de autopercepciones del desenvolvimiento en distintos contextos relacionales (la familia, la escuela, los pares del mismo sexo, los pares del otro sexo...). Parece preferible definirlo como la autopercepción de competencias que las personas activan transversalmente en la vida social (Infante et al., 2002; Yáñez, Valdés, \& Vera, 2012). Precisamente, para evitar ese reduccionismo, el cuestionario AUSO (Fernández-Zabala et al., 2016) contempla la dimensión de competencia social.

En todo caso, la competencia social no abarca todo el ámbito semántico de la persona en cuanto ser social. El desarrollo social hace referencia a procesos de socialización, tales como la formación de vínculos afectivos, la adquisición de los valores, las normas y los conocimientos sociales, el aprendizaje de costumbres, roles y conductas que la sociedad transmite y exige cumplir a cada uno de sus miembros (López, Etxebarria, Fuentes, \& Ortiz, 2001). La razón de diferenciar en el autoconcepto social una dimensión denominada responsabilidad social radica en el hecho de que el sentirse competente socialmente no agota la autopercepción del 
desarrollo social. Por tanto, las dimensiones que propone el cuestionario de Autoconcepto Social (AUSO; Fernández-Zabala et al., 2016) parecen más adecuadas que otras propuestas para tratar de dar cuenta del desarrollo social, en su sentido más amplio, en función de variables como el sexo y la edad.

El objetivo de esta investigación es doble: por un lado, comprobar la invarianza factorial entre hombres y mujeres y entre dos grupos de diferente edad con el propósito de asegurar que la estructura de medida es equivalente (Elosua \& Muñiz, 2010); y, por otro lado, precisar las diferencias en el autoconcepto social en función del sexo y la edad medidas con el Cuestionario de Autoconcepto Social (AUSO) de FernándezZabala (2011). Con ello, además de refrendar la calidad de las características psicométricas del AUSO, se busca completar las carencias de conocimiento (inconsistencias, desatención a dimensiones básicas del autoconcepto social) que han quedado expuestas.

\section{Método}

\section{Participantes}

Participan en esta investigación estudiantes de centros escolares públicos y privados de la Comunidad Autónoma del País Vasco (España), de un nivel sociocultural medio. Tras la depuración de 74 sujetos outliers, la muestra queda compuesta por 767 sujetos, 319 hombres (41.5\%) y 449 mujeres (58.5\%), con edades comprendidas entre los 16 y los 25 años $(\mathrm{M}=$ 19.97; DT = 1.86). Para comprobar diferencias de edad, los participantes se dividen en dos grupos: 465 menores de 20 años (60.6\%), 185 hombres $(39.8 \%)$ y 280 mujeres $(60.6 \%)$ y 302 mayores de 20 años (39.4\%), 133 hombres (44\%) y 169 mujeres (56\%).

\section{Instrumentos}

Para medir las autopercepciones sociales, se usa el Cuestionario de Autoconcepto Social (AUSO) de Fernández-Zabala (2011) compuesto por 8 ítems (cf. anexo 1): 4 de ellos corresponden a la escala de responsabilidad social que mide la percepción de cada cual sobre su contribución al funcionamiento social, al bien común y a la mejora de la humanidad; los otros 4 conforman la escala de competencia social que mide la autopercepción de las capacidades que cada cual activa en situaciones sociales y de cómo percibe la reacción de los demás hacia sí. La respuesta a los ítems se ofrece en una escala de tipo Likert con cinco opciones donde $1=$ Falso y 5 = Verdadero. El cuestionario cuenta con índices psicométricos adecuados (FernándezZabala et al., 2016): consistencia interna (alpha de Cronbach) de 0.77; coeficiente de fiabilidad compuesta Omega de McDonald $=0.904$ y la varianza media extractada $=0.546$, también adecuada. Por un lado, el coeficiente de fiabilidad compuesta indica la consistencia interna de los indicadores que conforman un factor; su valor no depende del número de ítems que conforman el factor, considerándose aceptable si $\mathrm{FC} \geq 0.70$. La varianza media extractada, por otro lado, indica el porcentaje de varianza del factor que ha sido capturada por el constructo, comparada con la varianza debida al error de medida, considerándose óptimos valores en torno a $\mathrm{VME}=0.50$ o superiores (Hair, Black, Babin, Anderson, \& Tatham, 2009).

\section{Procedimiento}

Tras contactar y lograr el consentimiento oportuno tanto de la dirección de los centros educativos colaboradores en este estudio como de los profesores y padres (en el caso de los participantes menores de edad), se procedió a la administración de los cuestionarios en horario lectivo y dentro de las aulas por los propios autores. A todos los participantes se les aseguró el anonimato de sus respuestas, a la vez que se evitó que conociesen la finalidad de la investigación con el fin de favorecer la sinceridad en sus respuestas y de prevenir, de este modo, la probabilidad del efecto de deseabilidad social. La 
cumplimentación del cuestionario no exige más de cinco minutos.

\section{Análisis de datos}

El tratamiento de los valores perdidos (0.92\%) se realiza mediante la opción de imputación múltiple que el programa LISREL 8.8 (Jöreskog \& Sörbom, 2006) ofrece a partir del algoritmo de maximización de expectativa (EM) y de la Cadena Markov Monte Carlo (MCMC, generación aleatoria de distribución de probabilidad vía cadenas de Markov). Esta opción permite extraer una puntuación aproximada a dicho ítem basada en las respuestas totales dadas por el propio sujeto.

El primer paso para llevar a cabo el contraste de la equivalencia factorial consiste en verificar por separado en las cuatro submuestras (hombres/mujeres, menores de 20 años/mayores de 20), la bondad del ajuste de la estructura bifactorial del AUSO mediante el análisis factorial confirmatorio. A continuación, se realiza el análisis progresivo de la invarianza comenzando por la invarianza configural e imponiendo secuencialmente restricciones más estrictas de forma que se analizan también la invarianza métrica (o también denominada débil), la fuerte y la estricta. Siguiendo la recomendación de Cheung y Rensvold (1999), se utilizó el cambio en el índice CFI menor o igual a 0.01 entre los sucesivos niveles de invarianza como punto de corte indicativo de que la invarianza del modelo se acepta.

Los análisis de diferencia de medias en las escalas del AUSO, en función de las variables independientes edad y sexo, así como el efecto de la interacción entre ambas, se realiza mediante un MANOVA de diseño 2 × 2 (sexo x grupo de edad) a partir del programa SPSS 20 para Windows (IBM Corp., 2012).

\section{Resultados}

Invarianza factorial en función del sexo y de la edad

En primer lugar, se comprueba el ajuste del modelo a los datos de forma independiente en cada una de las submuestras: hombres, mujeres, menores de 20 y mayores de 20 años. Los resultados obtenidos se presentan en la Tabla 1.

\section{TABLA 1}

Índices de bondad de ajuste en las diferentes muestras en función del sexo y de la edad

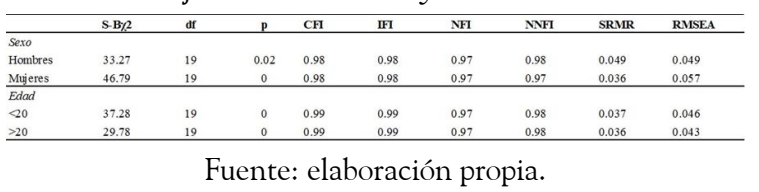

Todos los índices de bondad de ajuste extraídos en las cuatro submuestras resultan ser adecuados. Los valores del Comparative Fit Index (CFI), del Incremental Fit Index (IFI), Normed y NoNormed Fix Index (NFI y NNFI) se sitúan en todos los casos por encima del 0.95 requerido; en cuanto al índice Standardized Root Mean Residual (SRMR) presenta un valor inferior a 0.05, lo cual indica un buen ajuste. También el valor del índice Root Mean Square Error Aproximation (RMSEA) resulta ser adecuado al ser inferior a 0.06 , indicando nuevamente un buen ajuste del modelo tanto para la variable sexo como para la variable edad (Hu \& Bentler, 1999).

Una vez confirmada, por tanto, la estructura de medida en las cuatro submuestras, se procede a continuación a realizar el análisis de la equivalencia factorial que trata de conocer si dicha estructura se mantiene invariante en las submuestras en función del sexo y de la edad. Los datos obtenidos se presentan en la Tabla 2.

\section{TABLA 2}

Análisis de la invarianza según los indicadores absolutos y comparativos

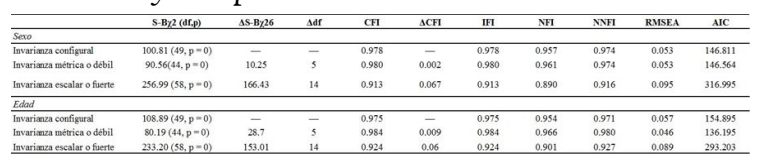

Fuente: elaboración propia. 
El primer paso consiste en comprobar la invarianza configural obligando a que la estructura factorial sea idéntica para todas las muestras elegidas (Sierra, Santos-Iglesias, \& Vallejo-Medina, 2012). Los resultados aportados por el análisis multigrupo permiten aceptar la equivalencia de los modelos de medida en las dos agrupaciones de la muestra (CFI, IFI, NFI y NNFI $>0.95$; RMSEA < 0.06). Por tanto, los modelos base para la prueba de invarianzas ajustan los datos y, en consecuencia, pueden ser aceptados como modelos estructuralmente equivalentes.

El segundo paso consiste en someter a prueba la invarianza métrica (o débil), añadiendo al modelo base las restricciones sobre los coeficientes regresores, de forma que se obliga a que las saturaciones de los ítems sobre su factor sean las mismas para todas las muestras. Los valores de los parámetros recogidos en la Tabla 2 permiten aceptar la invarianza métrica independientemente del sexo y la edad, ya que los índices de bondad se mantienen en unos rangos aceptables que apoyan la conclusión (CFI, IFI, NFI y NNFI > 0.95; RMSEA < 0.06). Además, el criterio de información de Akaike en la invarianza tanto sobre el sexo como sobre la edad sufre una reducción muy débil al incluir las restricciones propias de la invarianza débil, por lo que se mantiene casi invariante. En cuanto al segundo parámetro de comparación entre modelos (CFI), su incremento es mínimo con respecto a la invarianza anterior, no superando el diferencial de 0.01 estipulado.

En cambio, en ninguna de las dos invarianzas fuertes examinadas para la edad y el sexo que tratan de comparar la equivalencia de los modelos respecto de los valores interceptales, la raíz cuadrática media logra reducir el valor crítico de RMSEA $\leq$ 0.08. Tampoco los índices de bondad extraídos para cada una de las submuestras llegan al mínimo establecido (CFI, IFI, NFI y NNFI < 0.95). En cuanto al índice comparativo de Bentler, este es muy superior al límite fijado tanto para la variable sexo (CFIdébil-fuerte $=0.067$ ), como parar la variable edad $\left(\mathrm{CFI}_{\text {débil-fuerte }}=0.06\right)$, y el incremento en el índice de Akaike es considerable también para ambas variables $\left(\mathrm{AIC}_{\text {fuerte-débil }}=170.431\right.$;
$\left.\mathrm{AIC}_{\text {fuerte-débil }}=157.008\right)$. Por ello, puede afirmarse que no se dispone de suficiente apoyo empírico para poder aceptar el modelo de invarianza fuerte en ninguna de las dos submuestras.

No se analizó la invarianza estricta, debido a que supone añadir otra restricción más a las propias de la invarianza fuerte, de forma que ya, de base, nunca podría cumplirse al no cumplirse la restricción inicial anterior (Almeida et al., 2011).

\section{Diferencia de medias}

En la Tabla 3, se exponen los datos del contraste de medias y el tamaño del efecto en el AUSO.

TABLA 3

Efectos principales y de interacción de las variables sexo y edad sobre el autoconcepto social

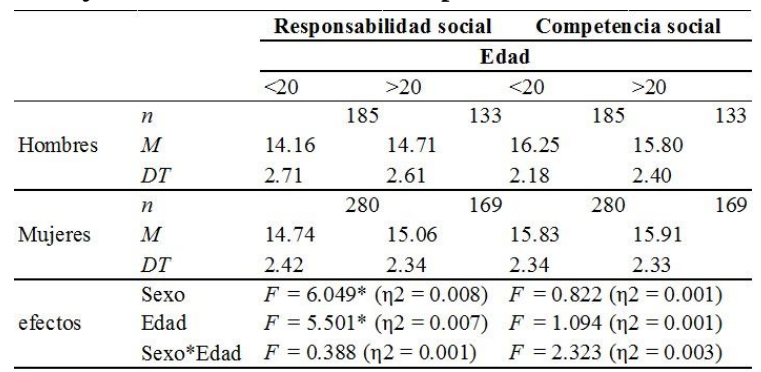

Nota: $\mathrm{p}<0.05$.

Fuente: Elaboración propia.

En la autopercepción de la competencia social, no se observan diferencias asociadas ni al sexo ni a la edad. En cambio, los resultados indican diferencias estadísticamente significativas en la escala de responsabilidad social con puntuaciones superiores de las mujeres con respecto a los hombres y de las personas de más de 20 años con respecto a las menores de ese corte de edad. No se comprueba, en cambio, un efecto de interacción significativa entre ambas variables, lo cual indica que en responsabilidad social los hombres obtienen mayores puntuaciones que las mujeres y los mayores de 20 años mejores puntuaciones que los menores de 20, pero el efecto de la edad no consigue que las mujeres igualen o superen la puntuación de los hombres en dicha variable. 
Analizando las estimaciones del grado en que cada variable (sexo y grupo de edad) o la combinación de dichas variables están afectando el autoconcepto social, se comprueba que ambas variables tienen un efecto pequeño en los dos componentes del autoconcepto social. Los índices $\eta 2$ obtenidos indican que la responsabilidad social resulta muy ligeramente mejor explicada por el sexo, mientras que en la competencia social la capacidad explicativa de las variables y de su interacción es muy baja, prácticamente nula.

\section{Discusión}

En este estudio, se aportan nuevos refrendos empíricos a la calidad psicométrica (FernándezZabala et al., 2016) del Cuestionario de Autoconcepto Social (AUSO) e información contrastada sobre el cambio de las autopercepciones sociales en función de la edad y del sexo.

Contar con un instrumento de medida de características psicométricas adecuadas, como el AUSO, permitirá ir precisando mejor la función del autoconcepto, y de cada uno de sus componentes, en el comportamiento humano.

Uno de los tópicos más frecuentes en los estudios psicológicos consiste en verificar eventuales diferencias asociadas a la edad y al sexo por tratarse de una información fundamental para la comprensión de cualquier característica psicológica. Ahora bien, para precisar tales diferencias, no basta con contrastar las puntuaciones obtenidas en las respuestas a un cuestionario obviando una constatación previa: que la estructura de medida es equivalente para los diversos grupos de participantes (Elosua \& Muñiz, 2010) o, dicho de otra forma, que el cuestionario mantiene invarianza factorial en las distintas muestras que se comparan.

En este estudio, se analizan de forma progresiva cuatro tipos de invarianza factorial del Cuestionario de Autoconcepto Social (AUSO) que permiten afirmar que la configuración bifactorial, y por tanto la estructura factorial del cuestionario, se mantiene estable independiente de la edad y del sexo. También la saturación de cada ítem sobre su propio factor es equivalente a parte del sexo y de la edad, de forma que, además de la estructura factorial, se asegura la estabilidad del peso que cada ítem presenta sobre la escala para la que fue construido. Los datos indican a su vez que las medias de los grupos varían según las dos variables analizadas. A partir de ahí, los datos de las comparaciones intergrupos indican que las medias de los grupos varían según las dos variables analizadas, asegurando a través de la invarianza configural y métrica que tales diferentes no son debidos a una estructura factorial del cuestionario diferente o a distintos pesos de los propios ítems. Conforme a los datos de este estudio, no se observan diferencias asociadas ni a la edad ni al sexo en la autopercepción de la competencia social. Esta constatación coincide en lo sustancial con lo previamente conocido sobre el autoconcepto social en cuanto competencia/aceptación social por más que se hayan observado diferencias específicas en la autopercepción de ciertos tipos de relación (por ejemplo con los pares del mismo o del otro sexo). Hubiese sido esperable que, por efectos del desarrollo psicológico, de las ayudas culturales y de la ampliación de relaciones sociales, los jóvenes de más de 20 años se percibiesen con mayor competencia/ aceptación social que los más jóvenes. Tal mejora se observa, además, en otros dominios del autoconcepto, como el físico (Goñi, Rodríguez, \& Ruiz de Azua, 2004). Dada la ausencia de esta evolución cabría, entonces, suponer que nuestro actual contexto socioeconómico y cultural no facilita precisamente el incremento de una autopercepción social positiva si bien no deja de ser una hipótesis de trabajo que precisará de futuras comprobaciones.

La evolución asociada a la edad y al sexo de la otra dimensión del autoconcepto, la autopercepción de la responsabilidad social, es diferente. En este caso, las mujeres se autoperciben con mayor implicación en la sociedad que los hombres a la vez que esa autopercepción mejora en el grupo mayor de 20 años con respecto al grupo de menor edad. Estos resultados apuntan a un mantenimiento de los 
patrones estereotipados de género socialmente aceptados según los cuales se espera de las chicas una mayor responsabilidad social (Gilligan, 1982). En la misma línea, en otros estudios, se ha encontrado que las mujeres informan de niveles más altos en la dimensión moral del autoconcepto (Goñi, 2009; Goñi, FernándezZabala, \& Infante, 2012). Por otro lado, el incremento significativo que experimentan los participantes de 20 años frente al grupo de menor edad es un claro ejemplo de la importancia que adquieren las relaciones sociales con los iguales durante esta etapa del ciclo vital.

Disponer de una visión precisa de las diversas dimensiones del autoconcepto social y de sus pautas de asociación con la edad y el sexo abre posibilidades a la intervención educativa, ya que dentro de una concepción jerárquica y multidimensional del autoconcepto, si bien el autoconcepto global es resistente a modificaciones, sus dimensiones concretas son susceptibles de mayor modificabilidad y, por tanto, precisarían de una intervención psicológica diferencial (Shavelson et al., 1976).

En todo caso, quedan pendientes de desarrollo investigaciones que pongan de relieve vinculaciones de la autopercepción tanto de competencia social como de responsabilidad social con otras variables sociopersonales, ya que identificar este tipo de conexiones resulta de gran valor al desvelar flancos por donde afrontar una intervención educativa que ayude a construir un mejor ajuste sociopersonal (RamosDíaz, Rodríguez-Fernández, Fernández-Zabala, Revuelta, \& Zuazagoitia, 2016; RodríguezFernández, Droguett, \& Revuelta, 2012; Rodríguez-Fernández, Ramos-Díaz, FernándezZabala, Goñi, Esnaola, \& Goñi, 2016).

Los resultados de este estudio precisan, de todos modos, de nuevos estudios dado que las franjas de edad sometidas a contraste no cubren ni con mucho todo el periodo vital y, por otro lado, falta por comprobarse diferencias en el autoconcepto social asociadas a otro amplio abanico de variables sociopersonales, tales como las habilidades sociales, el apoyo social o el estatus social.
Entre las variables psicológicas con las que mantiene relación sería interesante en particular analizar las relaciones entre la responsabilidad social entendida como dimensión del autoconcepto social y la dimensión moral del autoconcepto personal debido a que se trata, indudablemente, de dos dimensiones separadas relacionadas ambas con la vida social.

\section{Agradecimientos}

Los autores de este estudio son miembros del grupo Consolidado de Investigación IT934-16 del Sistema Universitario Vasco. El estudio se enmarca como parte de los proyectos de investigación PPG17/61 y EHUA15/15 de la Universidad del País Vasco.

\section{Referencias}

Almeida, L. S., Prieto, K. D., Ferreira, A., Ferrando, M., Ferrandiz, C., Bermejo, R., \& Hernández, D. (2011). Structural invariance of multiple intelligences, based on the level of execution. Psicothema, 23(4), 832-838.

Amezcua, J., \& Pichardo, M. (2000). Diferencias de género en autoconcepto en sujetos adolescentes [Teenagers' gender differences in self-concept]. Anales de Psicología, 16(2), 207-214. https://doi.org/10.6018/29421

Bowles, T. V. P., \& Fallon, B. J. (1996). Selfconcept, family functioning and problem type: A replication and extension of a study of clinic and non-clinic adolescents. Journal of Adolescent Health, 19, 62-67. https://doi. org/10.1016/1054-139X(95)00129-G

Bracken, B. (1992). The multidimensional Self Concept Scale. Austin, TX: Pro-Ed.

Chang, L., McBride-Chang, C., Stewart, S. M., $\&$ Au, E. (2003). Life satisfaction, selfconcept, and family relations in Chinese adolescents and children. International Journal of BehavioralDevelopment, 27(2), 182-189. https://doi.org/10.1080/01650250 244000182 
Cheung, G. W., \& Rensvold, R. B. (1999). Testing factorial invariance across groups: A reconceptualization and proposed new method. Journal of Management, 25, 1-27. http://dx.doi.org/10.1177/014920639 902500101

Costa, S., \& Tabernero, C. (2012). Rendimiento académico y autoconcepto en estudiantes de Educación Secundaria Obligatoria según el género [Academic achievement and self-concept in students of Secondary Education by gender]. Revista Iberoamericana de Psicología y Salud, 3(2), 175-193.

Elosua, P., \& Muñiz, J. (2010). Exploring the factorial structure of the self-concept: A sequential approach using CFA, MIMIC, and MACS models, across gender and two languages. EuropeanPsychologist, 15(1), 58-67. https://doi.org/10.1027/1016-9040/ a000006

Esnaola, I. (2005). Desarrollo del autoconcepto durante la adolescencia y principio de la juventud [Development of self-concept during adolescence and early youth]. Revista de Psicología General y Aplicada, 58(2), 265-277.

Esnaola, I., Infante, G., \& Zulaika, L. M. (2011). The multidimensional structure of physical self-concept. The Spanish Journal of Psychology, 14(1), 304-312. http://dx.doi.or g/10.5209/rev_SJOP.2011.v14.n1.27

Fernández-Zabala, A. (2011). El autoconcepto social en la adolescencia y juventud: Dimensiones, medida y relaciones [The social self-concept in adolescence and youth: Dimensions, measurement and relationships] (Tesis doctoral). Leioa, España: Servicio Editorial de la Universidad del País Vasco.

Fernández-Zabala, A., Rodríguez-Fernández, A., \& Goñi, A. (2016). La estructura del Cuestionario de Autoconcepto Social (AUSO) [The structure of the Social Selfconcept (SSC) Questionnaire]. Anales de Psicología, 32 (1), 199-205. http://dx.doi.org /10.6018/analesps.32.1.193931
Garaigordobil, M., Dura, A., \& Pérez, J. I. (2005). Síntomas psicopatológicos, problemas de conducta y autoconcepto-autoestima: un estudio con adolescentes de 14 a 17 años [Psychopathological symptoms, behavioural problems, and selfconcept-selfesteem: A study of adolescents aged 14 to 17 years old]. Anuario de Psicología Clínica y de la Salud, 1, 53-63.

García, F., \& Musitu, G. (2001). AF5: Autoconcepto Forma 5 [AF5: Self-concept form 5]. Madrid, España: TEA.

Gilligan, C. (1982). In a different voice: Psychological theory and women's development. Cambridge, Massachusetts: Harvard University.

Goñi, A., Rodríguez, A., \& Esnaola, I. (2010). Las autopercepciones físicas en la edad adulta y en la vejez [Physical selfperceptions in adulthood and old age]. Psicothema, 33(1), 27-34.

Goñi, A., Rodriguez, A., \& Ruiz de Azua, S. (2004). Bienestar psicológico y autoconcepto fisico en la adolescencia y juventud [Psychological well-being and physical self-concept in adolescence and youth]. Psiquis, 24(4), 17-27.

Goñi, E. (2009). El autoconcepto personal: Estructura interna, medida y variabilidad [The personal self-concept: Internal structure, measurement and variability] (Tesis doctoral). Leioa, España: Servicio Editorial de la Universidad del País Vasco.

Goñi, E., Fernández-Zabala, A., \& Infante, G. (2012). El autoconcepto personal: Diferencias asociadas a la edad y al sexo. Aula Abierta, 40(1), 39-50.

Goñi, E., Madariaga, J., Axpe, I., \& Goñi, A. (2011). Structure of the Personal SelfConcept (PSC) questionnaire. International Journal of Clinical and Health Psychology, 11(3), 509-522.

Hair, J. F., Black, W. C., Babin, B. J., Anderson, R. E., \& Tatham, R. L. (2009). Multivariate data analysis (7th edn). Upper Saddle River, NJ: Pearson Education.

Harter, S. (1982). The Perceived Competence Scale for Children. Child Development, 
53(1), 87-97. https://doi.org/10.2307/1129 640

Hu, L., \& Bentler, P. M. (1999). Cut-off criteria for fit indices in covariance structure analysis: Conventional criteria versus new alternatives. Structural Equation Modeling, 6, 1-55. https://doi.org/10.1080/107055199 09540118

IBM Corp. (2012). IBM SPSS Statistics for Windows, Version 20.0. Armonk, NY: IBM Corp.

Infante, L., De la Morena, L., García, B., Sánchez, A., Hierrezuelo, L., \& Muñoz, A. (2002). Un estudio sobre el autoconcepto social en estudiantes de E.S.O.: diferencias de género [A research of the social self-concept with high school students: Gender differences]. Revista Electrónica Interuniversitaria de Formación del Profesorado, 5(3), 1-7. Recuperado de http://www.revistaeducacion.educacion .es/re352/re352_22.pdf

Inglés, C. J., Pastor, Y., Torregrosa, M. S., Redondo, J., \& García, J. M. (2009). Diferencias en función del género y del curso académico en dimensiones del autoconcepto: estudio con una muestra de adolescentes españoles [Gender and grade level differences in self.concept dimensions: Study with Spanish adolescent simple]. Anuario de Psicología, 40 (2), 271-288.

Jöreskog, K. G., \& Sörbom, D. (2006). LISREL 8.8 for Windows [Computer software]. Lincolnwood, IL: Scientific Software International.

López, F., Etxebarria, I., Fuentes, M. J., \& Ortiz, M. J. (2001). Desarrollo afectivo y social [Emotional and social development]. Madrid, España: Pirámide.

Marsh, H. W. (1989). Age and sex effects in multiple dimension of self-concept: Preadolescence to early adulthood. Journal of Educational Psychology, 81, 417-430.

Musitu, G., García, F., \& Gutiérrez, M. (1991). Autoconcepto Forma A. Manual [Self-concept form A. Manual]. Madrid, España: TEA.

Pastor, Y., Balaguer, I., \& García-Merita, M. (2003). El autoconcepto y la autoestima en la adolescencia media: análisis diferencial por curso y género [Self-concept and self esteem in middle adolescence: Differential analysis by grade and gender]. Revista de Psicología Social, 18, 141-159. https://doi.or g/10.1174/021347403321645258

Peixoto, F., \& Almeida, L. S. (2011). Self-Concept organization: Analysis of hierarchical structure in adolescents. Psicología: Reflexão e Crítica, 24(3), 533-541. https://doi.org/10.1590/S0102-79 722011000300014

Ramos-Díaz, E., Rodríguez-Fernández, A., Fernández-Zabala, A., Revuelta, L. \& Zuazagoitia, A. (2016). Adolescent students \# perceived social support, selfconcept and school engagement. Revista de Psicodidáctica, 21(2), 339-356. doi: 10.1387/RevPsicodidact.14848

Rodríguez-Fernández, A., Droguett, L., \& Revuelta, L. (2012). School and personal adjustment in adolescence: The role of academic self-concept and perceived social support. Revista de Psicodidáctica, 17(2), 397-414. doi: 10.1387/Rev.Psicodidact.3002

Rodríguez-Fernández, A., Axpe, I., \& Goñi, A. (2015). Propiedades psicométricas de una versión abreviada del Cuestionario de Autoconcepto Físico (CAF) [Psychometric properties of of an abbreviated version of the Physical Self Questionnaire (CAF)]. Actas Españolas de Psiquiatría, 43(4), 125-132.

Rodríguez-Fernández, A., Ramos-Díaz, E., Fernández-Zabala, A., Goñi, E., Esnaola, I., \& Goñi, A. (2016). Contextual and psychological variables in a descriptive model of subjective well-being and school engagement. International Journal of Clinical and Health Psychology, 16(2), 166-174. doi: 10.1016/j.ijchp.2016.01003

Shavelson, R., Hubner, J., \& Stanton, J. (1976). Self-concept: Validation of construct interpretations. Review of Educational Research, 46(3), 407-441. https://doi.org/10 $.3102 / 00346543046003407$ 
Sierra, J. C., Santos-Iglesias, P., \& VallejoMedina, P. (2012). Evaluación de la equivalencia factorial y métrica de la Sexual Assertiveness Scale (SAS) por sexo [Evaluation of the factorial and metric equivalence of the Sexual Assertiveness Scale (SAS) by sex]. Psicothema, 24(2), 316-322.

Xu, M. K., Marsh, H. W., Hau, K.-T., Ho, T. I., Morin, A. J. S., \& Abduljabbar, A. S. (2013). The internal/external frame of reference of academic self-concept: Extension to a foreign language and the role of language of instruction. Jounal of Educational Psychology, 105(2), 489-503. doi: $10.1037 / \mathrm{a} 0031333$

Yáñez, A., Valdés, A., \& Vera, J. (2012). Caracterización del autoconcepto de estudiantes sobresalientes. En R. DíazLoving, S. Rivera, \& I. Reyes (Eds.), Aportaciones actuales de la psicología social (pp. 169-173). Monterrey: AMEPSO.

\section{Anexo A}

Ítems del Cuestionario de Autoconcepto Social (AUSO)

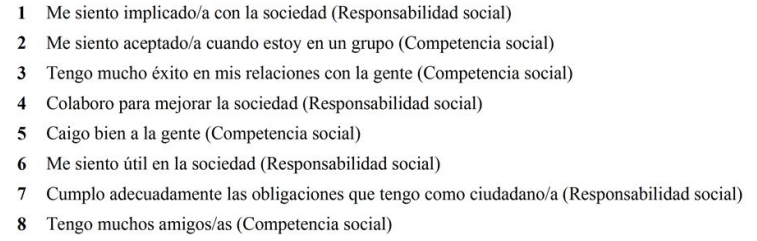

\section{Notas}

* Artículo de investigación 